\title{
Journal of Microbiology and Genetics
}

Kamata $\mathrm{H}$ and Matsuhashi M. J Microbiol Genet: JMGE-115

\section{Deletion of Lipoprotein Gene rlpA Causes Supersensitivity of Es- cherichia coli to Several Antibiotics}

\author{
Hideaki Kamata ${ }^{1,2^{*}}$, Michio Matsuhashi ${ }^{1,3}$ \\ ${ }^{1}$ Institute of Applied Microbiology, University of Tokyo, Bunkyo-ku, Tokyo, Japan \\ ${ }^{2}$ Department of Molecular Medical Science, Graduate School of Biomedical Science, Hiroshima University, Kasumi, Minami-ku, \\ Hiroshima, Japan \\ ${ }^{3}$ Department of Biological Science and Technology, School of High Technology for Human Welfare, Tokai University. Numazu City, \\ Shizuoka, Japan
}

"Corresponding author: Hideaki Kamata, Institute of Applied Microbiology, University of Tokyo, Bunkyo-ku, Tokyo, Japan. Tel: +812575138; Fax: +812575136; Email: hkamata@hiroshima-u.ac.jp

Citation: Kamata H, Matsuhashi M (2017) Deletion of Lipoprotein Gene rlpA Causes Supersensitivity of Escherichia coli to Several Antibiotics. J Microbiol Genet: JMGE-115. DOI: 10.29011/2574-7371.100015

Received Date: 02 November, 2017; Accepted Date: 10 November, 2017; Published Date: 17 November, 2017

\begin{abstract}
The Escherichia coli RlpA lipoprotein localizes to the septal ring and binds to peptidoglycan through the SPOR domain. We generated an rlpA deletion mutant. RlpA deletion mutant and double mutants lacking both RlpA and Lpp lipoproteins grew normally without significant changes in cell morphology. However, these mutants are supersensitive to several antibiotics such as moenomycin, macarbomycin, enramycin, vancomycin, and bacitracin.
\end{abstract}

Keywords: Antibiotics; Deletion mutant; Escherichia coli; Lipoprotein; RlpA

\section{Introduction}

Bacterial growth and morphology are regulated by peptide glycan synthesis complexes and associated membrane proteins [1]. The chromosomal region at $14 \mathrm{~min}$ on the Escherichia coli chromosome map (the mrd region) contains several genes involved in cell duplication and morphogenesis [2]. These include mrdA [3] (also called pbpA [4]), which codes for the penicillin-binding protein PBP-2; $\operatorname{mrdB}$ [3] (also called rodA [5]), which codes for the RodA protein; and dacA [6], which codes for PBP-5. PBP-2 and RodA are involved in determination of the rod shape of the $E$. coli cell, functioning in the biosynthesis of peptidoglycan during cell growth [7]. PBP-5 is a D-alanine carboxypeptidase involved in the maturation of peptidoglycan [6].

Previously, we identified two rare lipoprotein genes, rlpA and rlpB (also called lptE), in the mrd region [8]. RlpA is located between $\mathrm{mrdB}$ and dacA and encodes a $36-\mathrm{kDa}$ lipoprotein, while $\mathrm{rlpB} / \mathrm{lptE}$ is located several kilobases upstream from mrdA/pbp2 and encodes a 19-kDa lipoprotein. These lipoproteins localize to the Outer Membrane (OM), and their maturation is inhibited by globomycin, an inhibitor of signal peptidase for the major $E$. coli Lipoprotein (Lpp) and other lipoproteins $[9,10]$. Though rlpA and $\mathrm{rlpB} / \mathrm{lptE}$ appear to be expressed at low levels, their product lipoproteins may still be functionally important for the cell.

\section{Materials and Methods}

Recent studies have revealed that the RlpB/LptE lipoprotein forms the LptD- LptE complex, which is responsible for inserting lipopolysaccharide into the outer leaflet of the OM [11-15]. The RlpA lipoprotein localizes to the ring-shaped apparatus called the septal ring, which mediates bacterial cytokinesis by binding to septal peptide glycan through the C-terminal SPOR domain [1618]. The E. coli chromosome encodes four SPOR domain proteins, namely FtsN, DamX, DedD, and RlpA, all of which localize to the septal ring. Mutation studies have revealed that FtsN, DamX, and DedD are cell division proteins and are involved in bacterial cytokinesis [16-19]. However, there is no evidence as yet that RlpA is involved in cell division and morphogenesis. Thus, the physiological role of RlpA remains unknown. To elucidate the physiological function of RlpA, we constructed an RlpA deletion mutant through homologous recombination. Double mutants lacking both the RlpA and Lpp lipoproteins were also constructed, 


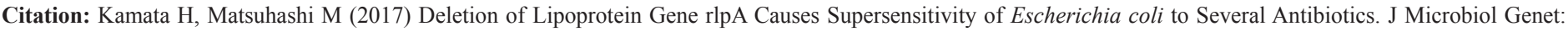
JMGE-115. DOI: 10.29011/2574-7371.100015

and the effect of deleting rlpA was determined.

The $E$. coli strains used in this study are listed in Table 1. For the genetic experiments and the antibiotic sensitivity test, modified Lennox-broth, L' broth, were used, supplemented with $10 \mathrm{~g} / \mathrm{L}$ polypeptone (Daigo Eiyo Chemical Co., Osaka), $5 \mathrm{~g} / \mathrm{L}$ yeast extract, $5 \mathrm{~g} / \mathrm{L} \mathrm{NaCl}, 1 \mathrm{~g} / \mathrm{L}$ glucose, $20 \mathrm{mg} / \mathrm{L}$ thymine, and $0.1 \mathrm{mg} / \mathrm{L}$ lipoic acid (L' agar). For the P1 phage transduction experiments, L'-agar plate containing $50 \mathrm{~m} \mathrm{~g} / \mathrm{ml}$ kanamycin, and M9 agar plates supplemented with $50 \mathrm{mg} / \mathrm{L}$ appropriate amino acids, $1 \mathrm{mg} / \mathrm{L}$ thiamine and $0.1 \mathrm{mg} / \mathrm{L}$ lipoic acid were used. $\mathrm{P} 1$ phage transduction technique was performed as previously described [20].

\begin{tabular}{|c|c|c|}
\hline Strains & Relevant properties* & References \\
\hline JE1011 & $\begin{array}{c}\text { F- thr leu trp his thi thy ara lac gal } \\
\text { xyl mtl rpsL tonA }\end{array}$ & M. Ishibash \\
\hline EC2009 & JE1011, DrlpA kmr.dir. & This study \\
\hline EC3001 & JE1011, DrlpA km.rev. & This study \\
\hline EC2001 & JE1011, DrlpA km.dir. dacA11191 & This study \\
\hline EC3002 & JE1011, DrlpA kmr.rev. dacA11191 & This study \\
\hline GRB19 & JE1011, lpp & Spratt and Stoker [4] \\
\hline BC2001 & GRB19, DrlpA kmr.dir. lpp & This study \\
\hline BC3001 & GRB19, DrlpA kmr.rev. lpp & This study \\
\hline BCA2001 & $\begin{array}{l}\text { GRB19, DrlpA km².dir. dacA11191 } \\
\text { lpp }\end{array}$ & This study \\
\hline BCA3001 & $\begin{array}{l}\text { GRB19, DrlpA kmr.rev. dacA11191 } \\
\text { lpp }\end{array}$ & This study \\
\hline AT1325 & $\begin{array}{c}\text { F-lip-9 thi-1 his-4 purB15 proA2 } \\
\text { mlt-1 } \\
\text { xyl-5 galK12 lacY1 str35 }\end{array}$ & $\begin{array}{c}\text { Taylor and Thoman } \\
{[26]}\end{array}$ \\
\hline $\begin{array}{l}{ }^{*} \mathrm{~km}^{\mathrm{r}} \cdot \mathrm{dir} \\
\text { directio }\end{array}$ & 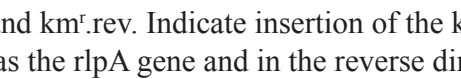 & $\begin{array}{l}\mathrm{m}^{\mathrm{r}} \text { gene in the same } \\
\text { ection, respectively. }\end{array}$ \\
\hline
\end{tabular}

Table 1: E. coli strains used in this study.

To construct the rlpA deletion mutant (DrlpA), we adopted a homologous recombination method, using a temperature-sensitive plasmid, as reported by Matsuyama et al. [21]. A 15-kb fragment of the E. coli dacA11191 [6] chromosome covering the region from leuS to dacA (mrd region) was cloned into the temperaturesensitive plasmid pMAN031. The rlpA gene was then replaced with a kanamycin resistance $\left(\mathrm{km}^{\mathrm{r}}\right)$ gene. The $\mathrm{km}^{\mathrm{r}}$ gene was inserted in each direction, resulting in the two plasmids pHK002 and $\mathrm{pHK} 003$, each of which carried $\mathrm{km}^{\mathrm{r}}$ in an opposite direction from the other (Figure 1(A)). E. coli JE1011 was transformed with these plasmids. Cells were then grown on L' agar plates containing
$50 \mu \mathrm{g} / \mathrm{mL}$ kanamycin, and were incubated overnight at $42^{\circ} \mathrm{C}$. The colonies were replicated on an L' agar plate containing $50 \mu \mathrm{g} / \mathrm{mL}$ ampicillin and incubated overnight at $30^{\circ} \mathrm{C}$. Five out of $10^{3} \mathrm{Km}^{\mathrm{r}}$ colonies were also determined to be ampicillin sensitive $\left(\mathrm{Amp}^{\mathrm{s}}\right)$. These $\mathrm{Km}^{\mathrm{r}} \mathrm{Amp}^{\mathrm{s}}$ colonies are believed to be mutants lacking the rlpA gene from the chromosome.
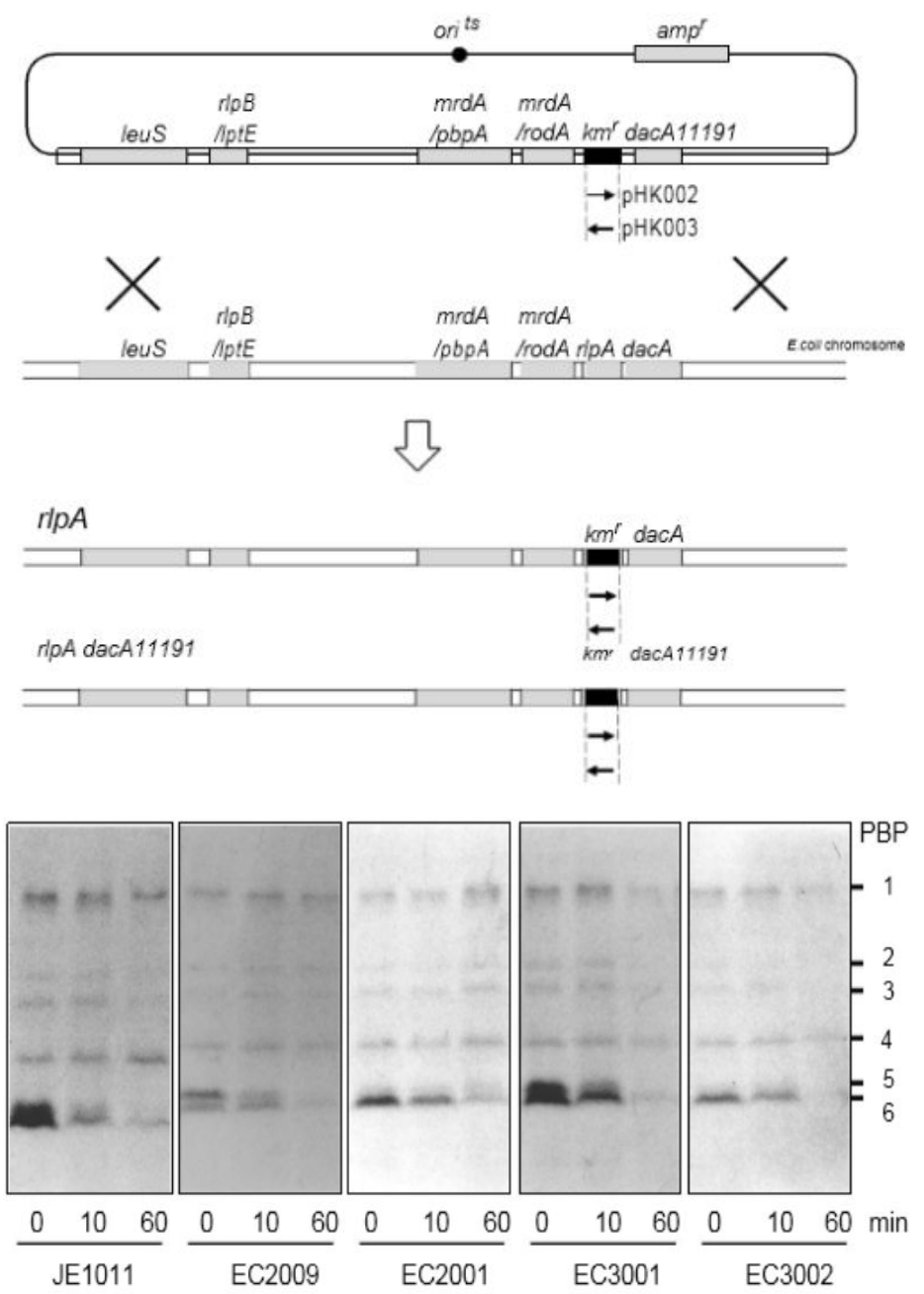

Figure 1: Isolation of DrlpA mutants by homologous recombination. (A) Homologous recombination of chromosome and plasmid led to deletion of the rlpA gene and insertion of the $\mathrm{km}^{\mathrm{r}}$ gene into the chromosome. Arrows indicate the direction of $\mathrm{km}^{\mathrm{r}}$ gene transcription. Two types of rlpA deletion mutants were obtained from transformants according to the sites of recombination, one deriving from recombination between $\mathrm{km}^{\mathrm{r}}$ and dacA and the other deriving from recombination between $\mathrm{km}^{\mathrm{r}}$ and dacA11191. (B) Chromosomal DNAs were digested with BamHI (left) and BgIII (right), and were subjected to southern blot hybridization with probe 1 (left) and probe 2 (right), respectively.

Since the plasmids pHK002 and pHK003 contained the dacA11191 mutant gene coding for a mutant PBP-5 with no penicillin-releasing activity [22]. We expected to obtain two types 


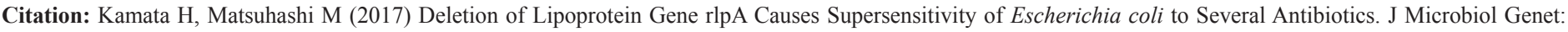
JMGE-115. DOI: 10.29011/2574-7371.100015

of mutants: DrlpA mutants with wild-type dacA and DrlpA dacA11191 mutants. Previously, we found that the E. coli dacA11191 mutant was more sensitive to penicillins than the wild-type dacA strain [3]. To distinguish between wild-type dac ${ }^{+}$strains and dacA11191 mutant strains, we measured the ampicillin sensitivities of the $\mathrm{Km}^{\mathrm{r}} \mathrm{Amp} \mathrm{p}^{\mathrm{s}}$ strains using a paper strip method. This allowed us to classify these strains as either ampicillin-sensitive or ampicillin-supersensitive. Two strains, E. coli EC2009 and EC3001, exhibited normal ampicillin sensitivity, whereas the other two strains, E. coli EC2001 and EC3002, exhibited ampicillin supersensitivity (Table 2). EC2001 and EC2009 were pHK002 transformants, and EC3001 and EC3002 were pHK003 transformants. We performed a penicillin-releasing assay [22] to confirm that the ampicillin-sensitive transformants expressed wild-type PBP-5, while the ampicillin-supersensitive transformants expressed the mutant PBP-5 (Figure 2)

\begin{tabular}{|c|c|c|c|c|c|c|c|c|c|c|}
\hline \multirow[t]{3}{*}{ Strains } & \multirow[t]{3}{*}{ Relevant properties } & \multicolumn{9}{|c|}{ Antibiotics } \\
\hline & & \multicolumn{8}{|c|}{ Paper strip method } & \multirow{2}{*}{$\begin{array}{l}\text { Step } \\
\text { dilution } \\
\text { method } \\
\text { Moeno- } \\
\text { Mycin } \\
\text { (MIC) }\end{array}$} \\
\hline & & Ampicillin & $\begin{array}{l}\text { Benzyl } \\
\text { Penicillin }\end{array}$ & $\begin{array}{l}\text { Moeno- } \\
\text { Mycin }\end{array}$ & $\begin{array}{l}\text { Macar- } \\
\text { Mycin }\end{array}$ & Enra-Mycin & $\begin{array}{l}\text { Vanco- } \\
\text { Mycin }\end{array}$ & $\begin{array}{l}\text { Baci- } \\
\text { Tracin }\end{array}$ & $\begin{array}{l}\text { Globo- } \\
\text { Mycin }\end{array}$ & \\
\hline JE1011 & & 4 & 4 & 1.2 & 0.5 & 0.5 & 1 & 0.5 & 4 & 50 \\
\hline EC2009 & JE1011, DrlpA km.dir. & 4 & 4.5 & 4.2 & 2.5 & 2 & 2 & 2 & 5 & 25 \\
\hline EC3001 & $\begin{array}{l}\text { JE1011, DrlpA kmr. } \\
\text { rev. }\end{array}$ & 4.5 & 4.5 & 4 & 3 & 2.5 & 2.5 & 2.5 & 5 & 25 \\
\hline EC2001 & $\begin{array}{c}\text { JE1011, DrlpA km.dir. } \\
\text { dacA11191 }\end{array}$ & 6 & 8 & 5.2 & 5 & 3.5 & 4 & 4 & 4.5 & 12.5 \\
\hline EC3002 & $\begin{array}{l}\text { JE1011, DrlpA kmr. } \\
\text { rev. dacA11191 }\end{array}$ & 7 & 7 & 5.5 & 5.2 & 4 & 3.8 & 3.5 & 4.8 & 12.5 \\
\hline GRB19 & lpp & 4 & 5.5 & 6 & 6.1 & 5 & 4 & 4 & 0.5 & 6.25 \\
\hline BC2001 & $\begin{array}{l}\text { GRB19, DrlpA kmr. } \\
\text { dir. lpp }\end{array}$ & 4 & 5.5 & 7 & 7.2 & 6 & 6 & 5 & 1 & 3.12 \\
\hline BC3001 & $\begin{array}{l}\text { GRB19, DrlpA km } \\
\text { rev. lpp }\end{array}$ & 4.2 & 5.5 & 7.5 & 7.2 & 6.4 & 5 & 5 & 1 & 3.12 \\
\hline BCA2001 & $\begin{array}{l}\text { GRB19, DrlpA km } \\
\text { dir. dacA11191 lpp }\end{array}$ & 6 & 7 & 8.5 & 8 & 7 & 6.8 & 6.5 & 1 & 1.56 \\
\hline BCA3001 & $\begin{array}{l}\text { GRB19, DrlpA km } \\
\text { rev. dacA11191 lpp }\end{array}$ & 6.5 & 7 & 8.5 & 8 & 7.5 & 6.8 & 6.8 & 1 & 1.56 \\
\hline \multicolumn{11}{|c|}{$\begin{array}{l}\text { For antibiotic sensitivity assay using the paper strip method, cell culture }\left(\mathrm{A}_{660}=0.1\right) \text { was diluted } 10 \text {-fold with } \mathrm{L}^{\prime} \text { broth without NaCl, and } 10 \propto \mathrm{L} \\
\text { aliquots were streaked onto L' plates without NaCl. Whatman No. } 3 \mathrm{MM} \text { filter paper strips }(0.3 \times 7.0 \mathrm{~cm}) \text { were moistened with solutions of solutions } \\
\text { of ampicillin }(0.2 \mathrm{mg} / \mathrm{ml}) \text {, penicillin }(0.2 \mathrm{mg} / \mathrm{ml}) \text {, moenomycin }(2.5 \mathrm{mg} / \mathrm{ml}) \text {, macarbomycin }(2.5 \mathrm{mg} / \mathrm{ml}) \text {, enramycin }(20 \mathrm{mg} / \mathrm{ml}) \text {, vancomycin }(20 \\
\mathrm{mg} / \mathrm{ml}) \text {, bacitracin }(20 \mathrm{mg} / \mathrm{ml}) \text { or globomycin }(2 \mathrm{mg} / \mathrm{ml}) \text {, and placed on the agar plates. E. coli were incubated for } 48 \mathrm{~h} \text { at } 42^{\circ} \mathrm{C} \text {. Values are expressed } \\
\text { as mm length of inhibitory zone. In the step dilution method, cells }\left(10^{4} / \mathrm{mL}\right) \text { were incubated in L' broth without NaCl containing serially diluted } \\
\text { moenomycin for } 48 \mathrm{~h} \text { at } 42^{\circ} \mathrm{C} \text {. Numbers show Minimum Inhibitory Concentrations (MIC) of moenomycin }(\mathrm{mg} / \mathrm{ml}) \text {. }\end{array}$} \\
\hline
\end{tabular}

Table 2: Estimation of antibiotic sensitivities of the E. coli $\mathrm{rlpA}^{+}$and $\Delta \mathrm{rlpA}$ strains. 


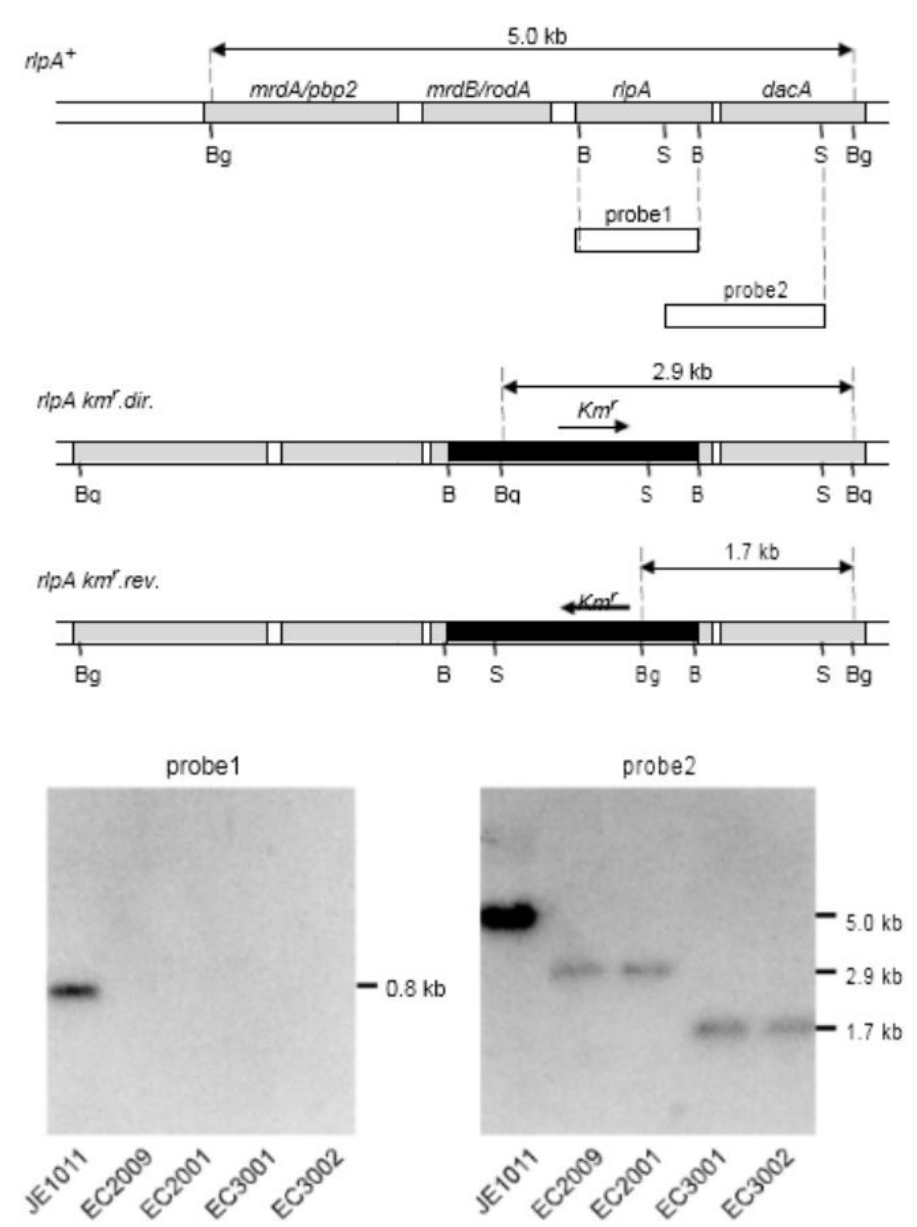

Figure 2: Penicillin-releasing assay of DrlpA mutants. Penicillin-binding proteins from the membrane fractions of $E$. coli strains were labeled with $\left[{ }^{14} \mathrm{C}\right]$ penicillin $\mathrm{G}$, and the release of penicillin was analyzed by addition of unlabeled penicillin G. After various lengths of time, the reaction was stopped, and the samples were subjected to SDS-PAGE and gel fluorograms.

The order of genes on the DrlpA mutant chromosome was determined by mapping of the $\mathrm{km}^{\mathrm{r}}$ gene with $\mathrm{P} 1$ phage and by Southern blot hybridization analysis. P1 phage transduction technique was performed as previously described [20]. DrlpA mutation was transduced with the $\mathrm{km}^{\mathrm{r}}$ gene as the marker using P1 phages grown in the DrlpA transformants into the recipient $E$.

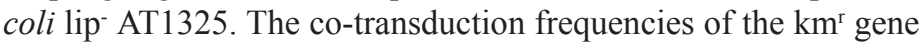
and the lip gene were $79 \%$ in EC2009, 80\% in EC3001, 78\% in EC2001, and 74\% in EC3002. These frequencies were similar to the co-transduction frequency $(76 \%)$ of $\mathrm{mrdB}$ and lip in a previous study [3]. Deletion of the rlpA gene from the E. coli chromosome was confirmed by Southern hybridization (Figure 1(B)).

Isolated DrlpA mutants grew normally in L' broth, exhibiting the normal rod shape. Omitting $\mathrm{NaCl}$ from the medium and growing cells at a higher temperature $\left(42^{\circ} \mathrm{C}\right)$ did not affect the growth or morphology of the DrlpA mutants. We detected no sign that the rlpA gene is indispensable for growth and shape-determination of the cell. Double mutant strains lacking both the RlpA and Lpp lipoproteins (BC2001 and BC3001) were isolated by transducing the lpp mutant strain $E$. coli GRB19 with P1 phage grown in one of the $E$. coli DrlpA mutants (EC2009 or EC3002) using the $\mathrm{km}^{\mathrm{r}}$ gene as a transduction marker. Triple mutants BCA2001 and BCA3001, carrying DrlpA lpp dacA11191, were isolated by transduction of $E$. coli GRB19 with $\mathrm{P} 1$ phage grown in E. coli EC2001 or EC3002. All mutants with both lipoprotein deletions exhibited the normal rod shape.

Despite exhibiting normal morphologies, the DrlpA mutants showed increased sensitivity to several antibiotics, as measured by paper strip and step dilution methods (Table 2). Vancomycin and bacitracin were obtained commercially from Sigma (St. Louis, MO, USA); ampicillin, benzylpenicillin, and macarbomycin were obtained from Meiji-Seika Co. (Tokyo, Japan); moenomycin was obtained from Hoechst (Frankfurt, Germany); enramycin was obtained from Takeda Chemical Industries (Osaka, Japan); and globomycin was obtained from Sankyo Co. (Tokyo, Japan). Sensitivity to antibiotics such as moenomycin, macarbomycin, enramycin, vancomycin, and bacitracin increased appreciably when the DrlpA mutation was introduced into the JE1011 strain, and sensitivity to globomycinalso increased slightly. In contrast, deletion of rlpA did not greatly affect sensitivity to penicillin, rifampicin, or novobiocin (data not shown). No increases in sensitivity to the above antibiotics have been observed due to introduction of the kanamycin resistance mutation alone (data not shown).

It is well known that SPOR-domain proteins are recruited to the septal ring, which mediates bacterial cytokinesis [1]. Among these proteins, FtsN is essential for cell division [19], and DamX and DedD are genuine division proteins that contribute significantly to the cell constriction process [16-18]. In contrast, the function of the SPOR-domain protein RlpA remains unclear. A recent study by Yahasiri et al. revealed that in Pseudomonas aeruginosa, RlpA is a lytic transglycosylase that contributes to rod shape and daughter cell separation [23]. Thus far, however, researchers have been unable to obtain any evidence indicating that the E. coli RlpA exhibits lytic transglycosylase activity [16]. We found that E. coli rlpA deletion mutants divided normally and did not show any significant morphological changes, consistent with previous findings [16-18].

\section{Conclusion}

Nevertheless, it is unlikely that E. coli RlpA is not involved in cell division or morphogenesis in some way. It has been reported that a truncated rlpA mutant is a multicopy suppressor of a mutation of the periplasmic protease gene prc, which is involved in cell division [24]. It is probable that double or multiple mutations in different lipoproteins are required to cause such phenotypic changes in the cell. Alternatively, RlpA may be 
Citation: Kamata H, Matsuhashi M (2017) Deletion of Lipoprotein Gene rlpA Causes Supersensitivity of Escherichia coli to Several Antibiotics. J Microbiol Genet: JMGE-115. DOI: 10.29011/2574-7371.100015

involved in the membrane integrity of E. coli. RlpA localizes not only to the septal ring but also to foci at various sites along the cell cylinder, suggesting that RlpA plays distinct roles in cytokinesis and envelope maturation [17]. Sensitivity to globomycin increased slightly in the DrlpA mutants, while it has been shown to decrease in lpp mutants [25]. As formation of the RlpA lipoprotein and its assembly at the membrane are inhibited by globomycin [8], the increased sensitivity of DrlpA mutants to globomycin may indicate that RlpA is involved in cell proliferation. In this model, RlpA would act as a minor component of membrane lipoproteins in the formation of the E. coli cell envelope.

\section{Acknowledgments}

We regret the early, sudden death of our collaborator Dr. Ichiro Takase. This article is dedicated to his memory.

\section{References}

1. Typas A, Banzhaf M, Gross CA, Vollmer W (2012) From the regulation of peptidoglycan synthesis to bacterial growth and morphology. Nat Rev Microbiol 10: 123-136.

2. Berlyn MK (1998) Linkage map of Escherichia coli K-12, edition 10: the traditional map. Microbiol Mol Biol Rev 62: 814-984.

3. Tamaki S, Matsuzawa H, Matsuhashi M (1980) Cluster of mrdA and mrdB genes responsible for the rod shape and mecillinam sensitivity of Escherichia coli. J Bacteriol 141: 52-57.

4. Spratt BG, Boyd A, Stoker N (1980) Defective and plaque-forming lambda transducing bacteriophage carrying penicillin-binding proteincell shape genes: genetic and physical mapping and identification of gene products from the lip-dacA-rodA-pbpA-leuS region of the Escherichia coli chromosome. J Bacteriol 143: 569-581.

5. Matsuzawa H, Hayakawa K, Sato T, Imahori K (1973) Characterization and genetic analysis of a mutant of Escherichia coli K-12 with rounded morphology. J Bacteriol 115: 436-442.

6. Matsuhashi M, Maruyama IN, Takagaki Y, Tamaki S, Nishimura Y, et al. (1978) Isolation of a mutant of Escherichia coli lacking penicillinsensitive D-alanine carboxypeptidase IA. Proc Natl Acad Sci USA 75: 2631-2635.

7. Ishino F, Park W, Tomioka S, Tamaki S, Takase I, et al. (1986) Peptidoglycan synthetic activities in membranes of Escherichia coli caused by overproduction of penicillin-binding protein 2 and $\operatorname{rodA}$ protein. $\mathrm{J}$ Biol Chem 261: 7024-7031.

8. Takase I, Ishino F, Wachi M, Kamata H, Doi M, et al. (1987) Genes encoding two lipoproteins in the leuS-dacA region of the Escherichia coli chromosome. J Bacteriol 169: 5692-5699.

9. Braun V, Rehn K (1969) Chemical characterization, spatial distribution and function of a lipoprotein (murein-lipoprotein) of the $E$. coli cell wall. The specific effect of trypsin on the membrane structure. Eur $\mathrm{J}$ Biochem 10: 426-438.

10. Ichihara S, Hussain M, Mizushima S (1981) Characterization of new membrane lipoproteins and their precursors of Escherichia coli. J Biol Chem 256: 3125-3129.
11. Dong H, Xiang Q, Gu Y, Wang Z, Paterson NG, et al. (2014) Structural basis for outer membrane lipopolysaccharide insertion. Nature 511: $52-56$.

12. Qiao S, Luo Q, Zhao Y, Zhang XC, Huang Y (2014) Structural basis for lipopolysaccharide insertion in the bacterial outer membrane. Nature 511: 108-111.

13. Chng SS, Xue M, Garner RA, Kadokura H, Boyd D, et al. (2012) Disulfide rearrangement triggered by translocon assembly controls lipopolysaccharide export. Science 337: 1665-1668.

14. Wu T, McCandlish AC, Gronenberg LS, Chng SS, Silhavy TJ, et al. (2006) Identification of a protein complex that assembles lipopolysaccharide in the outer membrane of Escherichia coli. Proc Natl Acad Sci U SA 103: 11754-11759.

15. Malojcic G, Andres D, Grabowicz M, George AH, Ruiz N, et al. (2014) LptE binds to and alters the physical state of LPS to catalyze its assembly at the cell surface. Proc Natl Acad Sci USA 111: 9467-9472.

16. Yahashiri A, Jorgenson MA, Weiss DS (2015) Bacterial SPOR domains are recruited to septal peptidoglycan by binding to glycan strands that lack stem peptides. Proc Natl Acad Sci USA 112: 11347-11352.

17. Gerding MA, Liu B, Bendezu FO, Hale CA, Bernhardt TG, et al. (2009) Self-enhanced accumulation of FtsN at Division Sites and Roles for Other Proteins with a SPOR domain (DamX, DedD, and RIpA) in Escherichia coli cell constriction. J Bacteriol 191: 7383-7401.

18. Arends SJ, Williams K, Scott RJ, Rolong S, Popham DL, et al. (2010) Discovery and characterization of three new Escherichia coli septal ring proteins that contain a SPOR domain: DamX, DedD, and RIpA. J Bacteriol 192: 242-255.

19. Dai K, Xu Y, Lutkenhaus J (1993) Cloning and characterization of ftsN an essential cell division gene in Escherichia coli isolated as a multicopy suppressor of ftsA12(Ts). J Bacteriol 175: 3790-3797.

20. Tamaki S, Nakagawa J, Maruyama I, Matsuhashi M (1979) Supersensitivity to beta-lactam antibiotics in Escherichia coli caused by D-alanine carboxypeptidase IA mutation. Agric Biol Chem 42: 2147-2150.

21. Matsuyama S, Mizushima S (1985) Construction and characterization of a deletion mutant lacking micF, a proposed regulatory gene for OmpF synthesis in Escherichia coli. J Bacteriol 162: 1196-1202.

22. Matsuhashi M, Tamaki S, Curtis SJ, Strominger JL (1979) Mutational evidence for identity of penicillin-binding protein 5 in Escherichia coli with the major D-alanine carboxypeptidase IA activity. J Bacteriol 137: 644-647.

23. Jorgenson MA, Chen Y, Yahashiri A, Popham DL, Weiss DS (2014) The bacterial septal ring protein RIpA is a lytic transglycosylase that contributes to rod shape and daughter cell separation in Pseudomonas aeruginosa. Mol Microbiol 93: 113-128.

24. Bass S, Gu Q, Christen A (1996) Multicopy suppressors of pre mutant Escherichia coli include two HtrA (DegP) protease homologs (HhoAB), DksA, and a truncated R1pA. J Bacteriol 178: 1154-1161.

25. Inukai M, Takeuchi M, Shimizu K, Arai M (1978) Mechanism of action of globomycin. J Antibiot (Tokyo) 31: 1203-1205.

26. Taylor AL, Thoman MS (1964) The Genetic Map of Escherichia coli K-12. Genetics 50: 659-677. 